\title{
Integrability of the Gibbons-Tsarev system
}

\author{
Maxim V. Pavlov \\ Department of Mathematical Physics, \\ P.N. Lebedev Physical Institute of Russian Academy of Sciences, \\ Moscow, Leninskij Prospekt, 53
}

\begin{abstract}
A new approach extracting multi-parametric hydrodynamic reductions for the integrable hydrodynamic chains is presented. The Benney hydrodynamic chain is considered.
\end{abstract}

\section{Contents}

1 Introduction $\quad 2$

2 Hamiltonian reductions $\quad 6$

3 Simplest solutions of the Gibbons-Tsarev system 11

4 Second and third particular cases 13

$\begin{array}{llr}5 & \text { Conclusion and Outlook } & 17\end{array}$

$\begin{array}{ll}\text { References } & 18\end{array}$ 
keywords: Poisson bracket, Hamiltonian structure, hydrodynamic chain, Liouville coordinates.

MSC: 35L40, 35L65, 37K10; $\quad$ PACS: 02.30.J, 11.10.E.

\section{Introduction}

The famous Benney hydrodynamic chain [1]

$$
A_{t}^{k}=A_{x}^{k+1}+k A^{k-1} A_{x}^{0}, \quad k=0,1, \ldots
$$

describes a fluid dynamics of a finite depth. This paper is dedicated to a construction of particular solutions.

1. Let us suppose that each moment $A^{k}$ can be decomposed on $N$ functions $f_{m(k)}(z)$ of a single variable ( $N$ is arbitrary), i.e.

$$
A^{k}=\sum_{m=1}^{N} f_{m(k)}\left(a^{m}\right)
$$

2. Let us substitute this ansatz (2) in (1) instead all moments $A^{k}$ with variable indices (except the first equation in (1)), and suppose that the summation with respect to the index $i$ can be removed from the hydrodynamic reduction

$$
\sum_{i=1}^{N}\left[f_{i(k)}^{\prime}\left(a^{i}\right) a_{t}^{i}-f_{i(k+1)}^{\prime}\left(a^{i}\right) a_{x}^{i}-k f_{i(k-1)}\left(a^{i}\right) A_{x}^{0}\right]=0, \quad k=1,2, \ldots, \quad i=1,2, \ldots, N .
$$

It means, that hydrodynamic type system

$$
a_{t}^{i}=\frac{f_{i(k+1)}^{\prime}\left(a^{i}\right)}{f_{i(k)}^{\prime}\left(a^{i}\right)} a_{x}^{i}+\frac{k f_{i(k-1)}\left(a^{i}\right)}{f_{i(k)}^{\prime}\left(a^{i}\right)} A_{x}^{0}, \quad k=1,2, \ldots, \quad i=1,2, \ldots, N
$$

cannot explicitly depend on the discrete variable $k$. A solution of the system

$$
b_{i}\left(a^{i}\right)=\frac{f_{i(k+1)}^{\prime}\left(a^{i}\right)}{f_{i(k)}^{\prime}\left(a^{i}\right)}, \quad c_{i}\left(a^{i}\right)=\frac{k f_{i(k-1)}\left(a^{i}\right)}{f_{i(k)}^{\prime}\left(a^{i}\right)}
$$

is given by

$$
f_{i(k)}\left(a^{i}\right)=\epsilon_{i} \frac{b_{i}^{k+1}\left(a^{i}\right)}{k+1}, \quad \frac{1}{c_{i}\left(a^{i}\right)}=b_{i}^{\prime}\left(a^{i}\right),
$$


where $\epsilon_{i}$ are arbitrary constants, and the functions $b_{i}(z)$ are not determined yet.

3. Let us suppose that $a^{i}$ are conservation law densities. Then $b_{i}(z) \equiv z$. The hydrodynamic reduction

$$
a_{t}^{i}=\left(\frac{\left(a^{i}\right)^{2}}{2}+u\right), \quad i=1,2, \ldots, N
$$

where $u=A^{0}(\mathbf{a})$, is the so-called "symmetric" hydrodynamic type system (see [11]) whose generating function of conservation laws

$$
p_{t}=\partial_{x}\left(\frac{p^{2}}{2}+u\right)
$$

can be obtained by the formal replacement $a^{i} \rightarrow p$.

Remark: The above construction was derived from the whole Benney hydrodynamic chain (1) excluding the first equation $A_{t}^{0}=A_{x}^{1}$. A substitution of the above formulae in this conservation law implies the important constraint

$$
\sum \epsilon_{m}=0
$$

4. A consistency of (4) with (3) implies the so-called Löwner equation (see [5] and [11])

$$
\partial_{i} p=\frac{\partial_{i} u}{a^{i}-p}\left(1+\sum \frac{\partial_{m} u}{a^{m}-p}\right)^{-1}
$$

where $\partial_{i} \equiv \partial / \partial a^{i}$. The compatibility condition $\partial_{i}\left(\partial_{k} p\right)=\partial_{k}\left(\partial_{i} p\right)$ leads to the GibbonsTsarev system (see [5] and [11])

$$
\left(a^{i}-a^{k}\right) \partial_{i k} u=\partial_{k} u \cdot \partial_{i} \delta u-\partial_{i} u \cdot \partial_{k} \delta u, \quad i \neq k,
$$

where $\delta \equiv \Sigma \partial / \partial a^{m}$. It is easy to see that the above moment decomposition (2)

$$
A^{0}(\mathbf{a})=\sum \epsilon_{m} a^{m}
$$

satisfies (7) without restriction (5).

Remark: Suppose that symmetric hydrodynamic type system (7) possesses the conservation law $u_{t}=v_{x}$ only (see $(1)$ ), where $v \equiv A^{1}(\mathbf{a})$. Then the compatibility condition 
$\partial_{i}\left(\partial_{k} v\right)=\partial_{k}\left(\partial_{i} v\right)$ implies $(7)$ again, where $\partial_{i} v=\left(a^{i}+\delta u\right) \partial_{i} u$. Thus, the existence of one extra conservation law leads to the existence of infinitely many conservation laws (see $(4))$.

5. Löwner equation (6) can be integrated once

$$
\lambda=p-\sum \epsilon_{m} \ln \left(p-a^{m}\right)
$$

under substitution (8). $\lambda$ is an integration constant here. Equation (9) of the Riemann surface (see [6], [7]) possesses an expansion $(\lambda \rightarrow \infty, p \rightarrow \infty)$

$$
\lambda=p+\frac{A^{0}}{p}+\frac{A^{1}}{p^{2}}+\frac{A^{2}}{p^{3}}+\ldots
$$

where all higher moments are determined by the moment decomposition (see (2), (5), cf. (8))

$$
A^{k}=\frac{1}{k+1} \sum_{m=1}^{N} \epsilon_{m}\left(a^{m}\right)^{k+1}, \quad k=0,1, \ldots
$$

Suppose that $\lambda$ is a function of $p$ and field variables $a^{k}$. Then the Vlasov (collisionless Boltzmann) equation (see [4], [10], [15])

$$
\lambda_{t}-p \lambda_{x}+\lambda_{p} u_{x}=0
$$

is fulfilled due to (1) and (10). A substitution of the inverse series

$$
p=\lambda-\frac{H_{0}}{\lambda}-\frac{H_{1}}{\lambda^{2}}-\frac{H_{2}}{\lambda^{3}}-\ldots
$$

in (10) implies explicit expressions for all conservation law densities $H_{k}\left(A^{0}, A^{1}, \ldots, A^{k}\right)$. For instance, $H_{0}=A^{0}, H_{1}=A^{1}, H_{2}=A^{2}+\left(A^{0}\right)^{2}, H_{3}=A^{3}+3 A^{0} A^{1}, \ldots$ A substitution (13) in (4) implies an infinite series of conservation laws

$$
\partial_{t} H_{n}=\partial_{x}\left(H_{n+1}-\frac{1}{2} \sum_{m=0}^{n-1} H_{m} H_{n-1-m}\right), \quad k=0,1, \ldots
$$

6. Hydrodynamic reductions (3) are semi-Hamiltonian (see [14]) hydrodynamic type systems which can be written in the diagonal form (see [5], [10])

$$
r_{t}^{i}=p^{i}(\mathbf{r}) r_{x}^{i}, \quad i=1,2, \ldots, N
$$


by virtue of invertible point transformations $a^{k}(\mathbf{r})$. Consistency of (15) with (4) leads to the Löwner equation $\left(\partial_{i} \equiv \partial / \partial r^{i}\right)$

$$
\partial_{i} p=\frac{\partial_{i} u}{p^{i}-p}
$$

written via Riemann invariants $r^{k}$ (cf. (6)) whose compatibility condition $\partial_{i}\left(\partial_{k} p\right)=$ $\partial_{k}\left(\partial_{i} p\right)$ implies the Gibbons-Tsarev system (cf. (7))

$$
\partial_{i} p^{k}=\frac{\partial_{i} u}{p^{i}-p^{k}}, \quad \partial_{i k} u=2 \frac{\partial_{i} u \cdot \partial_{k} u}{\left(p^{i}-p^{k}\right)^{2}}, \quad i \neq k
$$

originally derived in [5].

7. An arbitrary $N$ component hydrodynamic reduction (15) can be written in the combined form (see (3) and (14))

$$
\begin{aligned}
a_{t}^{k} & =\partial_{x}\left(\frac{\left(a^{k}\right)^{2}}{2}+H_{0}\right), \quad k=1,2, \ldots, K \\
\partial_{t} H_{n} & =\partial_{x}\left(H_{n+1}-\frac{1}{2} \sum_{m=0}^{n-1} H_{m} H_{n-1-m}\right), \quad n=0,1, \ldots, M-2, \\
\partial_{t} H_{M-1} & =\partial_{x}\left(H_{M}(\mathbf{a}, \mathbf{H})-\frac{1}{2} \sum_{m=0}^{M-2} H_{m} H_{M-2-m}\right)
\end{aligned}
$$

where $N=K+M$, and $H_{M}(\mathbf{a}, \mathbf{H})$ is some function determined from a consistency of the above hydrodynamic type system and the generating function of conservation laws (4), where $u \equiv H_{0}$.

Remark: A simplest choice $H_{M}(\mathbf{a}, \mathbf{H})=\Sigma \epsilon_{m} a^{m}$ extracts hydrodynamic reductions (17) equipped by local Hamiltonian structures of the Dubrovin-Novikov type (see the next Section).

Gibbons-Tsarev system (16) describes integrable hydrodynamic reductions (semiHamiltonian hydrodynamic type systems) of integrable Benney hydrodynamic chain (1). Thus, we believe that the Gibbons-Tsarev system is also integrable. At least, we know, that the Gibbons-Tsarev system possesses infinitely many solutions parameterized by $N$ 
arbitrary functions of a single variable (see [5]). All known solutions were found in [2], [4], [8], [9], [15]. Nevertheless, a construction of such solutions is an open problem now. By

this reason, we try to suggest the new approach allowing to seek most general families of particular solutions. This paper is devoted to a description of hydrodynamic reductions (17), where $H_{M}(\mathbf{a}, \mathbf{H})$ depends on all lower field variables $H_{n}(n=0,1, \ldots, M-1)$ and a sole function $\Delta=\Sigma f_{m}\left(a^{m}\right)$.

This paper is organized in the following way. In Section 2, hydrodynamic reductions equipped by local Hamiltonian structures of the Dubrovin-Novikov type are determined by the special choice $H_{M}=\Sigma \epsilon_{m} a^{m}$. In Section $3, N+2$ parametric family of solutions $u(\mathbf{a})$ of the Gibbons-Tsarev system is found for the first special sub-case $H_{0}=f_{0}(\Delta)$. A corresponding equation of the Riemann surface and corresponding two-parametric family of hydrodynamic chains are constructed. In Section 4, the second special sub-case $H_{1}=$ $f_{1}(\Delta)$ is considered. Corresponding hydrodynamic chain and the Riemann surface are presented. In Conclusion, a generalization of the approach presented in this paper is discussed.

\section{Hamiltonian reductions}

The Benney hydrodynamic chain belongs to the class of Egorov hydrodynamic chains (see details in [12], [13]), which possess a pair of conservation laws $a_{t}=b_{x}, b_{t}=c_{x}$. In the case of the Benney hydrodynamic chain, $a=H_{0}, b=H_{1}, c=H_{2}-H_{0}^{2} / 2$ (see (14)). All commuting flows of any integrable Egorov hydrodynamic chain are integrable Egorov hydrodynamic chains too. A generating function of such Egorov's pair is given by the so-called dispersionless Hirota equations (see, for instance, [3])

$$
\partial_{\tau(\zeta)} H_{0}=\partial_{x} p(\zeta), \quad \partial_{\tau(\zeta)} p(\lambda)=\partial_{x} \ln [p(\lambda)-p(\zeta)],
$$


where $p(\lambda)$ is a generating function of conservation law densities (see (4) and (13)) and $\partial_{\tau(\zeta)}$ is a formal operator (see, for instance, in [12]). Thus, the generating function of commuting hydrodynamic reductions (they are obtained by a formal replacement $p(\lambda) \rightarrow$ $a^{i}$ exactly as in (4))

$$
a_{\tau(\zeta)}^{i}=\partial_{x} \ln \left(p(\zeta)-a^{i}\right)
$$

possesses an infinite series of conservation laws (they can be obtained by a substitution of (13) in (18))

$\partial_{\tau(\zeta)} H_{0}=\partial_{x} p(\zeta), \quad \partial_{\tau(\zeta)} H_{1}=\partial_{x}\left(\frac{p^{2}(\zeta)}{2}+H_{0}\right), \quad \partial_{\tau(\zeta)} H_{2}=\partial_{x}\left(\frac{p^{3}(\zeta)}{3}+H_{0} p(\zeta)+H_{1}\right), \ldots$

Remark: A substitution of (13) for $p(\zeta)$ and the formal series

$$
\partial_{\tau(\zeta)}=-\frac{1}{\zeta} \partial_{t^{0}}-\frac{1}{\zeta^{2}} \partial_{t^{1}}-\frac{1}{\zeta^{3}} \partial_{t^{2}}-\ldots
$$

in (18) leads to an infinite series of generating functions (cf. (4))

$$
p_{t^{0}}=p_{x}, \quad p_{t^{1}}=\partial_{x}\left(\frac{p^{2}}{2}+H_{0}\right), \quad p_{t^{2}}=\partial_{x}\left(\frac{p^{3}}{3}+H_{0} p+H_{1}\right), \ldots
$$

A substitution of (13) for $p(\zeta)$ and (20) in (19) leads to the infinite series of commuting hydrodynamic reductions

$$
a_{t^{1}}^{k}=\partial_{x}\left(\frac{\left(a^{k}\right)^{2}}{2}+H_{0}(\mathbf{a})\right), \quad a_{t^{2}}^{k}=\partial_{x}\left(\frac{\left(a^{k}\right)^{3}}{3}+a^{k} H_{0}(\mathbf{a})+H_{1}(\mathbf{a})\right), \ldots
$$

Thus, the generalized hodograph method (established by S.P. Tsarev in [14] and adopted for an arbitrary set of commuting hydrodynamic type systems by J. Gibbons and Yu. Kodama in [6], [7]) implies an infinite series of particular solutions determined by the generating function

$x d a^{k}+t d\left(\frac{\left(a^{k}\right)^{2}}{2}+H_{0}(\mathbf{a})\right)+y d\left(\frac{\left(a^{k}\right)^{3}}{3}+a^{k} H_{0}(\mathbf{a})+H_{1}(\mathbf{a})\right)=d \ln \left(p-a^{k}\right), \quad k=1,2, \ldots, N$

for the Benney hydrodynamic chain as well as for the Khohlov-Zabolotzkaya system (see, for instance, [8])

$$
u_{t}=v_{x}, \quad u_{y}=v_{t}+u u_{x}
$$


where $x=t^{0}, t=t^{1}, y=t^{2}$.

Let us consider a set of hydrodynamic reductions (17) extracted by the constraint $H_{M}=\Sigma \epsilon_{m} a^{m}$.

1. In the case of the waterbag reduction (8), $H_{0}=\Sigma \epsilon_{m} a^{m}$. Thus, the simple computation (see (19))

$$
\partial_{\tau(\zeta)} H_{0} \equiv \partial_{\tau(\zeta)}\left(\sum \epsilon_{m} a^{m}\right)=\partial_{x}\left(\sum \epsilon_{m} \ln \left(p-a^{m}\right)\right)=\partial_{x} p(\zeta)
$$

leads to equation of the Riemann surface (9). The waterbag hydrodynamic reduction (see (3) and (9)) possesses the local Hamiltonian structure of the Dubrovin-Novikov type

$$
a_{t}^{i}=\frac{1}{\epsilon_{i}} \partial_{x} \frac{\partial H_{2}}{\partial a^{i}}
$$

where the momentum and the Hamiltonian densities are given, respectively, by

$$
H_{1}=\frac{1}{2} \sum \epsilon_{m}\left(a^{m}\right)^{2}, \quad H_{2}=\frac{1}{6} \sum \epsilon_{m}\left(a^{m}\right)^{3}+\frac{1}{2}\left(\sum \epsilon_{m} a^{m}\right)^{2} .
$$

2. Suppose, in comparison with the waterbag case, that $H_{1}=\Sigma \epsilon_{m} a^{m}$. Then (see (19))

$$
\partial_{\tau(\zeta)} H_{1} \equiv \partial_{\tau(\zeta)}\left(\sum \epsilon_{m} a^{m}\right)=\partial_{x}\left(\sum \epsilon_{m} \ln \left(p(\zeta)-a^{m}\right)\right)=\partial_{x}\left(\frac{p^{2}(\zeta)}{2}+H_{0}\right)
$$

leads to the equation of the Riemann surface

$$
\lambda=\frac{p^{2}}{2}+H_{0}-\sum \epsilon_{m} \ln \left(p-a^{m}\right)
$$

associated with $K+1$ component hydrodynamic type system

$$
a_{t}^{k}=\partial_{x}\left(\frac{\left(a^{k}\right)^{2}}{2}+H_{0}\right), \quad \partial_{t} H_{0}=\partial_{x}\left(\sum \epsilon_{m} a^{m}\right)
$$

which has the local Hamiltonian structure

$$
a_{t}^{i}=\frac{1}{\epsilon_{i}} \partial_{x} \frac{\partial H_{3}}{\partial a^{i}}, \quad \partial_{t} H_{0}=\partial_{x} \frac{\partial H_{3}}{\partial H_{0}},
$$

where the momentum and the Hamiltonian densities are given, respectively, by

$$
H_{2}=\frac{1}{2} \sum \epsilon_{m}\left(a^{m}\right)^{2}+\frac{1}{2} H_{0}^{2}, \quad H_{3}=\frac{1}{6} \sum \epsilon_{m}\left(a^{m}\right)^{3}+H_{0} \sum \epsilon_{m} a^{m}
$$


3. Suppose now, that $H_{2}=\Sigma \epsilon_{m} a^{m}$. Then (see (19))

$$
\partial_{\tau(\zeta)} H_{2} \equiv \partial_{\tau(\zeta)}\left(\sum \epsilon_{m} a^{m}\right)=\partial_{x}\left(\sum \epsilon_{m} \ln \left(p(\zeta)-a^{m}\right)\right)=\partial_{x}\left(\frac{p^{3}(\zeta)}{3}+H_{0} p(\zeta)+H_{1}\right)
$$

leads to the equation of the Riemann surface

$$
\lambda=\frac{p^{3}}{2}+H_{0} p+H_{1}-\sum \epsilon_{m} \ln \left(p-a^{m}\right),
$$

associated with $K+2$ component hydrodynamic type system

$$
a_{t}^{k}=\partial_{x}\left(\frac{\left(a^{k}\right)^{2}}{2}+H_{0}\right), \quad \partial_{t} H_{0}=\partial_{x} H_{1}, \quad \partial_{t} H_{1}=\partial_{x}\left(\sum \epsilon_{m} a^{m}-\frac{H_{0}^{2}}{2}\right)
$$

which has the local Hamiltonian structure

$$
a_{t}^{i}=\frac{1}{\epsilon_{i}} \partial_{x} \frac{\partial H_{4}}{\partial a^{i}}, \quad \partial_{t} H_{0}=\partial_{x} \frac{\partial H_{4}}{\partial H_{1}}, \quad \partial_{t} H_{1}=\partial_{x} \frac{\partial H_{4}}{\partial H_{0}}
$$

where the momentum and the Hamiltonian densities are given, respectively, by

$$
H_{3}=\frac{1}{2} \sum \epsilon_{m}\left(a^{m}\right)^{2}+H_{0} H_{1}, \quad H_{4}=\frac{1}{6} \sum \epsilon_{m}\left(a^{m}\right)^{3}+\frac{1}{2} H_{1}^{2}-\frac{1}{6} H_{0}^{3}+H_{0} \sum \epsilon_{m} a^{m} .
$$

All other hydrodynamic reductions of the Benney hydrodynamic chain possessing local Hamiltonian structures

$$
a_{t}^{i}=\frac{1}{\epsilon_{i}} \partial_{x} \frac{\partial H_{M+2}}{\partial a^{i}}, \quad \partial_{t} H_{k}=\partial_{x} \frac{\partial H_{M+2}}{\partial H_{M-k}}
$$

are associated with equations of the Riemann surfaces

$$
\lambda=\frac{p^{M+1}}{M+1}+H_{0} p^{M-1}+H_{1} p^{M-2}+P_{M-3}(\mathbf{H}, p)-\sum \epsilon_{m} \ln \left(p-a^{m}\right),
$$

where $P_{M-3}(\mathbf{H}, p)$ is a polynomial of a degree $M-3$ with respect to $p$, whose coefficients depend on $H_{0}, \ldots, H_{M-1}$. In such a case, the moment decomposition (cf. (11))

$$
\tilde{A}^{k}=\frac{1}{k+1-M} \sum_{m=1}^{N} \epsilon_{m}\left(a^{m}\right)^{k+1-M}, \quad k=M, M+1, \ldots
$$

reduces the Benney hydrodynamic chain

$$
\tilde{A}_{t}^{0}=\tilde{A}_{x}^{1}, \quad \tilde{A}_{t}^{k}=\tilde{A}_{x}^{k+1}+(k-M) \tilde{A}^{k-1} \tilde{A}_{x}^{0}, \quad k=1,2, \ldots
$$


to (21). These hydrodynamic reductions were found by the so-called "symmetry constraint" approach (see [2]). The moments $\tilde{A}^{k}$ are connected with $A^{k}$ by invertible triangular point transformations $\tilde{A}^{k}\left(A^{0}, A^{1}, \ldots A^{k}\right)$. It is easy to see comparing equations of the Riemann surfaces (cf. (10) and (22))

$$
\lambda=\frac{1}{M+1}\left(p+\sum_{k=0}^{\infty} \frac{A^{k}}{p^{k+1}}\right)^{M+1}=\frac{p^{M+1}}{M+1}+p^{M} \sum_{k=0}^{\infty} \frac{\tilde{A}^{k}}{p^{k+1}} .
$$

Remark: More complicated hydrodynamic reductions (cf. (17))

$$
\begin{aligned}
a_{t}^{k} & =\partial_{x}\left(\frac{\left(a^{k}\right)^{2}}{2}+H_{0}\right), \quad k=1,2, \ldots, K \\
\partial_{t} H_{n} & =\partial_{x}\left(H_{n+1}-\frac{1}{2} \sum_{m=0}^{n-1} H_{m} H_{n-1-m}\right), \quad n=0,1, \ldots, M-2, \\
\partial_{t} H_{M-1} & =\partial_{x}\left(H_{M}(\Delta, \mathbf{H})-\frac{1}{2} \sum_{m=0}^{M-2} H_{m} H_{M-2-m}\right),
\end{aligned}
$$

where $\Delta=\Sigma f_{k}\left(a^{k}\right)$, can be associated with different Riemann mappings $\lambda(p)$. Vlasov equation (12) is invariant under an arbitrary scaling $\tilde{\lambda}(\lambda)$. Let us consider in such a case, the substitution (instead of (10))

$$
\lambda=f\left(p+\frac{A^{0}}{p}+\frac{A^{1}}{p^{2}}+\frac{A^{2}}{p^{3}}+\ldots\right)
$$

where $f(p)$ is an arbitrary entire function. A corresponding Taylor expansion

$$
\lambda=f(p)+\frac{1}{p}\left(A^{0}+\frac{A^{1}}{p}+\frac{A^{2}}{p^{2}}+\ldots\right) f^{\prime}(p)+\frac{1}{2 p^{2}}\left(A^{0}+\frac{A^{1}}{p}+\frac{A^{2}}{p^{2}}+\ldots\right)^{2} f^{\prime \prime}(p)+\ldots
$$

can be reduced to the form

$$
\lambda=f(p)+\tilde{f}(p) \cdot\left(\tilde{A}^{0}+\frac{\tilde{A}^{1}}{p}+\frac{\tilde{A}^{2}}{p^{2}}+\ldots\right)
$$

where $\tilde{A}^{k}\left(A^{0}, A^{1}, \ldots, A^{k}\right)$ are polynomials, and $\tilde{f}(p)$ is some function. Indeed, a substitution of this ansatz in the Vlasov equation leads to the Benney hydrodynamic chain written in the form $\tilde{A}_{t}^{k}=\tilde{A}_{x}^{k+1}-\left[\beta_{-1} \tilde{A}^{k+1}+\beta_{0} \tilde{A}^{k}+\left(\beta_{1}+1-k\right) \tilde{A}^{k-1}+\beta_{2} \tilde{A}^{k-2}+\ldots+\beta_{k} \tilde{A}^{0}+\alpha_{k}\right] \frac{\tilde{A}_{x}^{0}}{\alpha_{-1}+\beta_{-1} \tilde{A}^{0}}$, 
where

$$
\frac{f^{\prime}(p)}{\tilde{f}(p)}=\alpha_{-1} p+\alpha_{0}+\frac{\alpha_{1}}{p}+\frac{\alpha_{2}}{p^{2}}+\ldots, \quad \frac{\tilde{f}^{\prime}(p)}{\tilde{f}(p)}=\beta_{-1} p+\beta_{0}+\frac{\beta_{1}}{p}+\frac{\beta_{2}}{p^{2}}+\ldots
$$

If, for instance, $f^{\prime}(p)=p^{M}$, then the Benney hydrodynamic chain $\left(\alpha_{-1}=1, \beta_{1}=M-1\right.$; all other coefficients $\alpha_{k}$ and $\beta_{k}$ vanish) can be written in form (24). We believe that all moments $\tilde{A}^{k}$ (except first $M$ moments $\tilde{A}^{0}, \tilde{A}^{0}, \ldots, \tilde{A}^{M-1}$ ) in the Benney hydrodynamic chain written in the above form can be expressed via field variables a as (cf. (2))

$$
\tilde{A}^{k}=\sum_{m=1}^{N} f_{m(k)}\left(a^{m}\right), \quad k=M, M+1, \ldots,
$$

for some set of constants $\alpha_{k}$ and $\beta_{k}$, where the functions $f_{m(k)}(z)$ are uniquely determined by the sole function $f(z)$ (see (26)). Indeed, a substitution of this ansatz in (24) immediately implies (23). Several such sub-cases are considered in next two Sections.

\section{Simplest solutions of the Gibbons-Tsarev system}

The first non-trivial case in this paper is given by $N$ component hydrodynamic reduction (3), where $u$ is a solution of Gibbons-Tsarev system (7). Let us suppose that $u$ depends

on a sole function $\Delta=\Sigma f_{k}\left(a^{k}\right)$ only. In such a case, three distinguished sub-cases can be extracted.

I. Gibbons-Tsarev system (7) admits $N$ parametric solution (8). This sub-case is well known, and already is considered with constraint (5) in previous Sections. Let us briefly consider this sub-case without constraint (5). Since a solution of the linear PDE (12) is determined up to an arbitrary function $\tilde{\lambda}(\lambda)$, let us choose a new function $\lambda$ such that $($ see $(9))$

$$
\lambda-\epsilon \ln \lambda=p-\sum \epsilon_{m} \ln \left(p-a^{m}\right)
$$

where $\epsilon \equiv \Sigma \epsilon_{m}$. A substitution of (13) instead $\lambda$ in the above formula leads to explicit expressions of conservation law densities $H_{n}$ via moments $A^{k}$ (see (11)). Then the Benney 
hydrodynamic chain

$$
A_{t}^{0}=\partial_{x}\left(A^{1}+\epsilon A^{0}\right), \quad A_{t}^{k}=A_{x}^{k+1}+k A^{k-1} A_{x}^{0}, \quad k=1,2, \ldots
$$

is equipped by the deformed Kupershmidt-Manin Poisson bracket (cf. [9])

$$
\left\{A^{0}, A^{0}\right\}=\epsilon \delta^{\prime}\left(x-x^{\prime}\right), \quad\left\{A^{k}, A^{n}\right\}=\left[k A^{k+n-1} \partial_{x}+n \partial_{x} A^{k+n-1}\right] \delta\left(x-x^{\prime}\right), \quad k+n>0 .
$$

II. Let us look for a solution of Gibbons-Tsarev system (7) in the more general form $u=\Sigma f_{m}\left(a^{m}\right)$, where $f_{k}\left(a^{k}\right)$ are not yet determined functions. A substitution of this ansatz in (7) leads to the new $N+1$ parametric solution

$$
u=\frac{1}{\epsilon_{0}} \sum \epsilon_{m} e^{\epsilon_{0} a^{m}}
$$

where $\epsilon_{0}$ and $\epsilon_{k}$ are arbitrary constants. A corresponding equation of the Riemann surface is given by

$$
\lambda=-\frac{1}{\epsilon_{0}} e^{-\epsilon_{0} p}-\sum \epsilon_{m} \int e^{-\epsilon_{0}\left(p-a^{m}\right)} d \ln \left(p-a^{m}\right) .
$$

Hydrodynamic type system (3) can be rewritten as the Benney hydrodynamic chain written in the form

$$
A_{t}^{k}=A_{x}^{k+1}+\left(\epsilon_{0} A^{k}+k A^{k-1}\right) A_{x}^{0}
$$

where

$$
d A^{k}=\sum \epsilon_{m}\left(a^{m}\right)^{k} e^{\epsilon_{0} a^{m}} d a^{m} .
$$

Then (28) (instead (10)) reduces to the form $(p \rightarrow \infty)$

$$
\lambda=e^{-\epsilon_{0} p}\left(-\frac{1}{\epsilon_{0}}+\sum_{m=0}^{\infty} \frac{A^{n}}{p^{n+1}}\right) .
$$

III. Let us look for a solution of Gibbons-Tsarev system $(7)$ in the form $u(\Delta)$, where $\Delta=\Sigma f_{m}\left(a^{m}\right)$, where $f_{k}\left(a^{k}\right)$ are not yet determined functions. A substitution of this ansatz in (7) leads to the new $N+2$ parametric solution

$$
u=\frac{1}{\sigma} \ln (\sigma \Delta+\xi), \quad f_{k}^{\prime}\left(a^{k}\right)=\epsilon_{k} \exp \left(\epsilon_{0} a^{k}-\frac{\sigma\left(a^{k}\right)^{2}}{2}\right)
$$


where $\sigma, \xi, \epsilon_{0}$ and $\epsilon_{k}$ are arbitrary constants (if $\sigma \neq 0$, the constant $\xi$ is removable by an appropriate shift $A^{0} \rightarrow A^{0}-\xi / \sigma$, i.e. just $N+2$ independent arbitrary constants). A corresponding equation of the Riemann surface can be found in quadratures

$$
d \lambda=(\sigma \Delta+\xi) e^{\frac{\sigma}{2} p^{2}-\epsilon_{0} p} d p-e^{\frac{\sigma}{2} p^{2}-\epsilon_{0} p} \sum \epsilon_{m} e^{\epsilon_{0} a^{m}-\frac{\sigma}{2}\left(a^{m}\right)^{2}} d \ln \left(p-a^{m}\right) .
$$

Let us introduce an infinite set of moments $A^{k}(\mathbf{a})$ determined by quadratures

$$
d A^{k}=\sum \epsilon_{m}\left(a^{m}\right)^{k} e^{\epsilon_{0} a^{m}-\frac{\sigma}{2}\left(a^{m}\right)^{2}} d a^{m} .
$$

In such a case, hydrodynamic type system can be written as the Benney hydrodynamic chain (cf. (1) and (14)) written in the form

$$
A_{t}^{k}=A_{x}^{k+1}+\left(-\sigma A^{k+1}+\epsilon_{0} A^{k}+k A^{k-1}\right) \frac{A_{x}^{0}}{\sigma A^{0}+\xi} .
$$

Then above equation of the Riemann surface reduces to the form $(p \rightarrow \infty)$

$$
\lambda=\xi \int e^{\frac{\sigma}{2} p^{2}-\epsilon_{0} p} d p+e^{\frac{\sigma}{2} p^{2}-\epsilon_{0} p} \sum_{n=0}^{\infty} \frac{A^{n}}{p^{n+1}} .
$$

\section{Second and third particular cases}

In this Section, two extra multi-parametric generalizations (see (25)) of hydrodynamic reductions equipped by local Hamiltonian structures of the Dubrovin-Novikov type (see Section 2) are described.

1. $K+1$ component hydrodynamic reduction (cf. (3) and (17))

$$
u_{t}=v_{x}, \quad a_{t}^{k}=\left(\frac{\left(a^{k}\right)^{2}}{2}+u\right), \quad k=1,2, \ldots, K
$$

where $v \equiv H_{1}(\mathbf{a}, u)$, is a semi-Hamiltonian hydrodynamic type system if at least one extra conservation law (see (14), $n=1$ )

$$
v_{t}=\partial_{x}\left(w-\frac{u^{2}}{2}\right)
$$


where $w \equiv H_{2}(\mathbf{a}, u)$, exists. In such a case, the compatibility conditions $\partial_{i}\left(\partial_{k} w\right)=\partial_{k}\left(\partial_{i} w\right)$ and $\partial_{k}\left(\partial_{u} w\right)=\partial_{u}\left(\partial_{k} w\right)$ lead to the Gibbons-Tsarev system (cf. (7))

$$
\begin{aligned}
\left(a^{i}-a^{k}\right) \partial_{i k} v & =\partial_{k} v \cdot \partial_{i u} v-\partial_{i} v \cdot \partial_{k u} v, i \neq k, \\
\partial_{k} v \cdot \partial_{u u} v & =\left(\partial_{u} v-a^{k}\right) \partial_{k u} v+\partial_{k} \delta v,
\end{aligned}
$$

where $\partial_{u} w=\delta v+\left(\partial_{u} v\right)^{2}+u$ and $\partial_{i} w=\left(\partial_{u} v+a^{i}\right) \partial_{i} v$. A consistency of (4) and (29) leads to the Löwner equations (cf. (6))

$$
\partial_{i} p=\frac{\partial_{i} v}{a^{i}-p}\left(p-\partial_{u} v+\sum \frac{\partial_{m} v}{a^{m}-p}\right)^{-1}, \quad \partial_{u} p=-\left(p-\partial_{u} v+\sum \frac{\partial_{m} v}{a^{m}-p}\right)^{-1} .
$$

Suppose (like in the previous Section) that $v$ depends on $u$ and a sole function $\Delta=$ $\Sigma f_{k}\left(a^{k}\right)$ only. This ansatz reduces the above Gibbons-Tsarev system to

$$
\partial_{\Delta \Delta} v=0, \quad \partial_{u u} v=\left(\partial_{u} v-a^{k}\right) \frac{\partial_{u \Delta} v}{\partial_{\Delta} v}+\frac{f_{k}^{\prime \prime}\left(a^{k}\right)}{f_{k}^{\prime}\left(a^{k}\right)}
$$

whose solution is given by

$$
v=(\beta \Delta+\alpha) e^{-\epsilon_{0} u}+\frac{\gamma}{\epsilon_{0}} u, \quad \ln f_{k}^{\prime}=\ln \epsilon_{k}+\gamma a^{k}-\frac{\epsilon_{0}}{2}\left(a^{k}\right)^{2}
$$

where $\alpha, \beta, \gamma, \epsilon_{0}$ and $\epsilon_{k}$ are constants.

Then these Löwner equations can be integrated once, and equation of the Riemann surface can be found in quadratures

$$
d \lambda=\frac{1}{\epsilon_{0}} d e^{\frac{\epsilon_{0}}{2} p^{2}-\gamma p+\epsilon_{0} u}+\epsilon_{0}(\beta \Delta+\alpha) e^{\frac{\epsilon_{0}}{2} p^{2}-\gamma p} d p-\beta e^{\frac{\epsilon_{0}}{2} p^{2}-\gamma p} \sum f_{m}^{\prime}\left(a^{m}\right) d \ln \left(p-a^{m}\right) .
$$

Introducing moments $A^{k}$ by their derivatives

$$
d A^{k}=\sum\left(a^{m}\right)^{k-1} f_{m}^{\prime}\left(a^{m}\right) d a^{m}, \quad k=1,2, \ldots,
$$

the above equation reduces to

$$
\lambda=\frac{1}{\epsilon_{0}} e^{\frac{\epsilon_{0}}{2} p^{2}-\gamma p+\epsilon_{0} u}+\alpha \epsilon_{0} \int e^{\frac{\epsilon_{0}}{2} p^{2}-\gamma p} d p+\beta e^{\frac{\epsilon_{0}}{2} p^{2}-\gamma p} \sum_{n=1}^{\infty} \frac{A^{n}}{p^{n}} .
$$


A corresponding form of the Benney hydrodynamic chain is given by

$$
\begin{aligned}
& u_{t}=\partial_{x}\left(\left(\beta A^{1}+\alpha\right) e^{-\epsilon_{0} u}+\frac{\gamma}{\epsilon_{0}} u\right), \\
& A_{t}^{k}=A_{x}^{k+1}+\left(-\epsilon_{0} A^{k+1}+\gamma A^{k}+(k-1) A^{k-1}\right) u_{x}, \quad k=1,2, \ldots
\end{aligned}
$$

Remark: Hydrodynamic reductions (29) depend on $N+3$ arbitrary parameters $\alpha, \beta, \epsilon_{0}$ and $\epsilon_{k}$, because the constant $\gamma$ can be removed by the Galilean transformation.

2. $K+2$ component hydrodynamic reduction (cf. (3) and (17))

$$
u_{t}=v_{x}, \quad v_{t}=\partial_{x}\left(w-\frac{u^{2}}{2}\right), \quad a_{t}^{k}=\left(\frac{\left(a^{k}\right)^{2}}{2}+u\right), \quad k=1,2, \ldots, K,
$$

where $w \equiv H_{2}(\mathbf{a}, u, v)$, is a semi-Hamiltonian hydrodynamic type system if at least one extra conservation law (see (14), $n=2$ )

$$
w_{t}=\partial_{x}(s-u v)
$$

where $s \equiv H_{3}(\mathbf{a}, u, v)$, exists. In such a case, the compatibility conditions $\partial_{i}\left(\partial_{k} s\right)=$ $\partial_{k}\left(\partial_{i} s\right), \partial_{k}\left(\partial_{u} s\right)=\partial_{u}\left(\partial_{k} s\right), \partial_{k}\left(\partial_{v} s\right)=\partial_{v}\left(\partial_{k} s\right), \partial_{v}\left(\partial_{u} s\right)=\partial_{u}\left(\partial_{v} s\right)$ lead to the GibbonsTsarev system (cf. (7))

$$
\begin{aligned}
\left(a^{i}-a^{k}\right) \partial_{i k} w & =\partial_{k} w \cdot \partial_{i v} w-\partial_{i} w \cdot \partial_{k v} w, i \neq k, \\
\partial_{k} w \cdot \partial_{v v} w & =\left(\partial_{v} w-a^{k}\right) \partial_{k v} w+\partial_{k u} w, \\
\partial_{u u} w+\partial_{v} w \cdot \partial_{u v} w & =\left(\partial_{u} w-u\right) \partial_{v v} w+\partial_{v} \delta w, \\
a^{k} \partial_{k u} w+\partial_{k} w \cdot \partial_{u v} w & =\left(\partial_{u} w-u\right) \partial_{k v} w+\partial_{k} \delta w,
\end{aligned}
$$

where $\partial_{u} s=v+\delta w+\left(\partial_{u} w-u\right) \partial_{v} w, \partial_{v} s=u+\partial_{u} w+\left(\partial_{v} w\right)^{2}$ and $\partial_{i} s=\left(\partial_{v} w+a^{i}\right) \partial_{i} w$. A 
consistency of (4) and (30) leads to the Löwner equations (cf. (6))

$$
\begin{aligned}
\partial_{i} p & =\frac{\partial_{i} w}{a^{i}-p}\left(p^{2}-p \partial_{v} w+u-w_{u}+\sum \frac{\partial_{m} w}{a^{m}-p}\right)^{-1}, \\
\partial_{u} p & =\left(\partial_{v} w-p\right)\left(p^{2}-p \partial_{v} w+u-w_{u}+\sum \frac{\partial_{m} w}{a^{m}-p}\right)^{-1}, \\
\partial_{u} p & =-\left(p^{2}-p \partial_{v} w+u-w_{u}+\sum \frac{\partial_{m} w}{a^{m}-p}\right)^{-1} .
\end{aligned}
$$

Suppose that $w$ depends on $u, v$ and a sole function $\Delta=\Sigma f_{k}\left(a^{k}\right)$ only. This ansatz reduces the above Gibbons-Tsarev system to

$$
\begin{aligned}
& \partial_{u \Delta} w=\partial_{\Delta} w \cdot \partial_{v v} w, \quad \partial_{u u} w+\partial_{v} w \cdot \partial_{u v} w=\left(\partial_{u} w-u\right) \partial_{v v} w, \\
& \partial_{\Delta \Delta} w=0, \quad \partial_{v \Delta} w=0, \quad \partial_{u v} w+a^{k} \frac{\partial_{u \Delta} w}{\partial_{\Delta} w}=\frac{f_{k}^{\prime \prime}\left(a^{k}\right)}{f_{k}^{\prime}\left(a^{k}\right)},
\end{aligned}
$$

whose solution is given by

$$
\begin{aligned}
w & =(\beta \Delta+\alpha) e^{-\epsilon_{0} u}-\frac{\epsilon_{0}}{2} v^{2}+(\gamma u+\delta) v+\frac{\epsilon_{0}-\gamma^{2}}{2 \epsilon_{0}} u^{2}+\left(-\frac{1+\gamma \delta}{\epsilon_{0}}+\frac{\gamma^{2}}{\epsilon_{0}^{2}}\right) u, \\
\ln f_{k}^{\prime} & =\ln \epsilon_{k}+\gamma a^{k}-\frac{\epsilon_{0}}{2}\left(a^{k}\right)^{2},
\end{aligned}
$$

where $\alpha, \beta, \gamma, \epsilon_{0}$ and $\epsilon_{k}$ are constants.

Then these Löwner equations can be integrated once, and equation of the Riemann surface can be found in quadratures

$$
\begin{aligned}
d \lambda= & d\left[\left(v+\frac{p-\gamma u-\delta}{\epsilon_{0}}+\frac{\gamma}{\epsilon_{0}^{2}}\right) e^{\frac{\epsilon_{0}}{2} p^{2}-\gamma p+\epsilon_{0} u}\right]+\epsilon_{0}(\beta \Delta+\alpha) e^{\frac{\epsilon_{0}}{2} p^{2}-\gamma p} d p \\
& -\beta e^{\frac{\epsilon_{0}}{2} p^{2}-\gamma p} \sum f_{m}^{\prime}\left(a^{m}\right) d \ln \left(p-a^{m}\right) .
\end{aligned}
$$

Introducing moments $A^{k}$ by their derivatives

$$
d A^{k}=\sum\left(a^{m}\right)^{k-2} f_{m}^{\prime}\left(a^{m}\right) d a^{m}, \quad k=2,3, \ldots
$$


the above equation reduces to

$$
\lambda=\left(v+\frac{p-\gamma u-\delta}{\epsilon_{0}}+\frac{\gamma}{\epsilon_{0}^{2}}\right) e^{\frac{\epsilon_{0}}{2} p^{2}-\gamma p+\epsilon_{0} u}+\alpha \epsilon_{0} \int e^{\frac{\epsilon_{0}}{2} p^{2}-\gamma p} d p+\beta e^{\frac{\epsilon_{0}}{2} p^{2}-\gamma p} \sum_{n=2}^{\infty} \frac{A^{n}}{p^{n-1}} .
$$

A corresponding form of the Benney hydrodynamic chain is given by

$$
\begin{aligned}
& u_{t}=v_{x}, \quad v_{t}=\partial_{x}\left(w-u^{2} / 2\right) \\
& A_{t}^{k}=A_{x}^{k+1}+\left(\epsilon_{0} A^{k+1}+\gamma A^{k}+(k-2) A^{k-1}\right) u_{x}, \quad k=2,3, \ldots,
\end{aligned}
$$

where

$$
w=\left(\beta A^{2}+\alpha\right) e^{-\epsilon_{0} u}-\frac{\epsilon_{0}}{2} v^{2}+(\gamma u+\delta) v+\frac{\epsilon_{0}-\gamma^{2}}{2 \epsilon_{0}} u^{2}+\left(-\frac{1+\gamma \delta}{\epsilon_{0}}+\frac{\gamma^{2}}{\epsilon_{0}^{2}}\right) u .
$$

Remark: Hydrodynamic reductions (30) depend on $N+4$ arbitrary parameters $\alpha, \beta, \gamma, \epsilon_{0}$ and $\epsilon_{k}$, because the constant $\delta$ can be removed by a combination of a shift and the Galilean transformation.

\section{Conclusion and Outlook}

In the same way, all other hydrodynamic reductions (25) can be completely described. Possibly, most general ansatz is given by (17), where the function $H_{K}(\mathbf{a}, \mathbf{H})$ depend on a finite number of moments $\tilde{A}^{l}$ (see $(27)$ ), i.e. $l=0,1, \ldots, L$, where $L$ is some natural number. For instance, let us suppose that $H_{0}$ depends on $\Delta_{1}=\Sigma f_{k(1)}\left(a^{k}\right)$ and $\Delta_{2}=\Sigma f_{k(2)}\left(a^{k}\right)$

only, and $f_{i}\left(a^{k}\right)$ are unknown functions yet. In the simplest case, $f_{k(1)}\left(a^{k}\right)=\epsilon_{k} a^{k}$ and $f_{k(2)}\left(a^{k}\right)=\epsilon_{k}\left(a^{k}\right)^{2}$. Corresponding hydrodynamic reduction is determined by $H_{0}=\left[\Delta_{2}-\right.$ $\left.\Delta_{1}^{2} /\left(1+\Sigma \epsilon_{m}\right)\right] / 2$.

Two important observations are made in this paper. The Löwner equations can be derived faster via generating functions of commuting flows (see, for instance, Section 2) than directly from a consistency of generating functions of conservation laws and corresponding "symmetric" hydrodynamic reductions. The Gibbons-Tsarev system can be 
derived faster from an existence of an extra conservation law than from a consistency of the Löwner equations. It looks like that this is a common feature of any integrable hydrodynamic chains. However, this, possibly, universal behavior needs a priori known formulation of hydrodynamic chains in their conservative form as well as generating functions of commuting flows, that is usually can be found just after a special separate investigation.

\section{Acknowledgement}

I thank Eugeni Ferapontov, John Gibbons, Andrea Raimondo, Vladimir Sokolov and Sergey Tsarev for their stimulating and clarifying discussions.

I am grateful to the Institute of Mathematics in Taipei (Taiwan) where some part of this work has been done, and especially to Jen-Hsu Chang for hospitality in National Defense University. This research was particularly supported by the Russian-Taiwanese grant 95WFE0300007 (RFBR grant 06-01-89507-HHC).

\section{References}

[1] D.J. Benney, Some properties of long non-linear waves, Stud. Appl. Math., 52 (1973) 45-50.

[2] L.V. Bogdanov, B.G. Konopelchenko, Symmetry constraints for dispersionless integrable equations and systems of hydrodynamic type, Phys. Lett. A, 330 (2004) 448-459.

[3] R. Carroll, Yu. Kodama, Solutions of the dispersionless Hirota equations, J. Phys. A: Math. Gen. 28 (1995) 6373-6387.

[4] J. Gibbons, Collisionless Boltzmann equations and integrable moment equations, Physica D, 3 (1981) 503-511. 
[5] J. Gibbons, S.P. Tsarev, Reductions of the Benney equations, Phys. Lett. A 211 (1996) 19-24. J. Gibbons, S.P. Tsarev, Conformal maps and reductions of the Benney equations, Phys. Lett. A 258 (1999) 263-271.

[6] J. Gibbons, Yu. Kodama, Integrable quasilinear systems: generalized hodograph transformation. Nonlinear evolutions (Balaruc-les-Bains, 1987), 97-107, World Sci. Publ., Teaneck, NJ, 1988. Yu. Kodama, J. Gibbons, A method for solving the dispersionless KP hierarchy and its exact solutions. II. Phys. Lett. A 135 (1989), No. 3, 167-170. Yu. Kodama, J. Gibbons, Integrability of the dispersionless KP hierarchy. Nonlinear world, Vol. 1 (Kiev, 1989), 166-180, World Sci. Publ., River Edge, NJ, 1990. J. Gibbons, L.A. Yu, The initial value problem for reductions of the Benney equations, Inverse Problems 16 No. 3 (2000) 605-618, L.A. Yu, Waterbag reductions of the dispersionless discrete KP hierarchy, J. Phys. A: Math. Gen., 33 (2000) $8127-8138$.

[7] Yu. Kodama, A method for solving the dispersionless KP equation and its exact solutions. Phys. Lett. A, 129 No. 4 (1988) 223-226. Yu. Kodama, A solution method for the dispersionless KP equation, Prog. Theor. Phys. Supplement. 94 (1988) 184. Yu. Kodama, Solutions of the dispersionless Toda equation, Phys. Lett. A, 147, No. 8-9 (1990) 477-482. Yu. Kodama, Exact solutions of hydrodynamic type equations having infinitely many conserved densities, Phys. Lett. A, 135 No. 3 (1989) 171-174.

[8] I.M. Krichever, The averaging method for two-dimensional "integrable" equations, Funct. Anal. Appl. 22 No. 3 (1988) 200-213, I.M. Krichever, Spectral theory of two-dimensional periodic operators and its applications, Russian Math. Surveys 44 No. 2 (1989) 145-225.

[9] B.A. Kupershmidt, Yu.I. Manin, Long wave equations with a free surface. I. Conservation laws and solutions. (Russian) Func. Anal. Appl. 11 No. 3 (1977), 31-42. 
B.A. Kupershmidt, Yu.I. Manin, Long wave equations with a free surface. II. The Hamiltonian structure and the higher equations. (Russian) Func. Anal. Appl. 12 No. 1 (1978), 25-37.

[10] A. Odesskii, M.V. Pavlov, V.V. Sokolov, A classification of integrable Vlasov-like equations. to appear in TMPh. 2008.

[11] M. V. Pavlov, Algebro-geometric approach in the theory of integrable hydrodynamic type systems. Comm. Math. Phys. 272 No. 2 (2007) 469-505.

[12] M. V. Pavlov, Classification of the Egorov hydrodynamic chains. Theor. Math. Phys. 138 No. 1 (2004) 55-71.

[13] M. V. Pavlov, S.P. Tsarev, Three-Hamiltonian structures of the Egorov hydrodynamic type systems, Funct. Anal. Appl., 37 No. 1 (2003) 32-45. M. V. Pavlov, Integrability of the Egorov hydrodynamic type systems, Theor. Maths. Phys., 150 No. 2 (2007) 225-243.

[14] S.P. Tsarev, On Poisson brackets and one-dimensional Hamiltonian systems of hydrodynamic type, Soviet Math. Dokl., 31 (1985) 488-491. S.P. Tsarev, The geometry of Hamiltonian systems of hydrodynamic type. The generalized hodograph method, Math. USSR Izvestiya 37 No. 2 (1991) 397-419.

[15] V.E. Zakharov, Benney's equations and quasi-classical approximation in the inverse problem method, Funct. Anal. Appl., 14 No. 2 (1980) 89-98. V.E. Zakharov, On the Benney's Equations, Physica 3D (1981) 193-200. 